\title{
XMM-Newton observation of PG 0844+349
}

\author{
W. Brinkmann ${ }^{1}$, D. Grupe ${ }^{2}$, G. Branduardi-Raymont ${ }^{3}$, and E. Ferrero ${ }^{2}$ \\ ${ }^{1}$ Centre for Interdisciplinary Plasma Science, Max-Planck-Institut für extraterrestrische Physik, Postfach 1312, \\ 85741 Garching, Germany \\ 2 Max-Planck-Institut für extraterrestrische Physik, Postfach 1312, 85741 Garching, Germany \\ 3 Mullard Space Science Laboratory, University College London, Holmbury St Mary, Dorking, Surrey, RH5 6NT, UK
}

Received 6 May 2002 / Accepted 4 November 2002

\begin{abstract}
In a $20 \mathrm{ksec}$ XMM-Newton observation the X-ray transient radio-quiet quasar PG 0844+349 was found in a historically high state compared to previous X-ray observations. The quasar showed a featureless spectrum with a strong soft excess over the extrapolation of a hard power law. Comptonization models or a broken power law with $\Gamma_{\text {soft }} \sim 2.75, \Gamma_{\text {hard }} \sim 2.25$ and a break energy of $E_{\text {break }} \sim 1.35 \mathrm{keV}$ represent acceptable descriptions of the spectral continuum. In the Comptonization models the temperature of the Comptonizing gas is considerably lower than generally found in (broad line) Seyfert galaxies whereas the optical depth is much higher. As a similar behavior has been seen in NLSy1 galaxies, it might be an indicator of the different physical conditions in these two classes of AGN. During the XMM-Newton observation the flux of PG 0844+349 varied achromatically in a smooth, nearly linear fashion, by $\sim 25 \%$ on time scales of a few thousand seconds, which puts some constraints on current models of Comptonizing accretion disk coronae.
\end{abstract}

Key words. galaxies: active - quasars: individual: PG 0844+349 - X-rays: galaxies

\section{Introduction}

Quasars as a class of Active Galactic Nuclei (AGN) exhibit a vast diversity in their observed properties. Some of these, like the radio-loud - radio-quiet dichotomy, can be related to the physical characteristics of the accreting central black hole and to morphological differences of the host galaxies. Others can result from specific geometrical conditions of the accretion flow and from the particular viewing condition of the observer with respect to the quasar (see, for example, Elvis 2000). In many cases, however, the causes for the unusual properties of a quasar remain obscure and thus the study of objects displaying atypical characteristics might yield important clues to the physical processes governing the quasar emission.

One of the objects with several extraordinary properties is PG $0844+349$, a well studied nearby $(z=0.064)$, bright $\left(m_{v}=14.0\right)$, radio-quiet quasar from the Palomar Green sample (Schmidt \& Green 1983). Deep radio observations reveal no nuclear source and the faint detection in the field $\left(S_{1.49 \mathrm{GHz}}=\right.$ $0.3 \mathrm{mJy}$ ) might be related to an $R=20 \mathrm{mag}$ object southwest of the quasar (Condon et al. 1987). PG 0844+349 is optically quite spectacular: it appears as a barred spiral with complex outer structure and a nearly equally bright companion galaxy (Hutchings \& Crampton 1990). It belongs to the class of AGN with unusually strong Fe II emission (Wang et al. 1996) and according to the optical spectrum displayed in

Send offprint requests to: W. Brinkmann, e-mail: wpb@rzg.mpg.de
Boroson \& Green (1992) the source shows all features of a Narrow-Line Seyfert 1 galaxy (NLSy1) - strong FeII emission and weak forbidden narrow lines, although its $F W H M(\mathrm{H} \beta)=$ $2420 \mathrm{~km} \mathrm{~s}^{-1}$ is slightly above the often used cut-off line of $2000 \mathrm{~km} \mathrm{~s}^{-1}$ (e.g. Osterbrock \& Pogge 1985). However, this cut-off for the classification of NLSy1s is more artificial than physical, so we still might define this source as a NLSy1 galaxy especially as it shows X-ray properties typical for this class of objects, i.e., a steep soft X-ray spectrum and strong variability (for reviews see Boller et al. 2000). It is infrared-loud with a disturbed host galaxy (Clements 2000), and it is strongly variable at all wavelengths on various time scales. The optical flux seems to have changed by $\sim 70 \%$ between 1986 (Elvis et al. 1994) and 1993 (Maoz et al. 1994) and intra-night variations with amplitudes of $\Delta B=0.08$ mag were detected by Jang et al. (1997). From reverberation measurements Kaspi et al. (2000) deduced a mass of $\sim 2.5 \times 10^{7} M_{\odot}$ for the quasar.

Compared to the ROSAT All Sky Survey (Yuan et al. 1998) the soft X-ray flux of PG $0844+349$ had decreased by a factor of $\sim 6$ in a pointed ROSAT observation 6 months later, without any noticeable changes in the spectral slope (Rachen et al. 1996). During the X-ray low state PG $0844+349$ had an Xray loudness of $\alpha_{\mathrm{ox}}>2$, i.e. it could be regarded as " $\mathrm{X}$-ray weak" compared to the average $<\alpha_{\text {ox }}>\sim 1.6$ for radio-quiet quasars (Yuan et al. 1998). In an ASCA observation the object was found in a high state, with a photon index of $\Gamma=1.98$ and a Fe $\mathrm{K} \alpha$ line with $E W \sim 300 \mathrm{eV}$. On shorter time scales, the $\mathrm{X}$-ray flux in the $2-10 \mathrm{keV}$ band is highly variable; the fastest 
variation detected is $60 \%$ in less than $2 \times 10^{4} \mathrm{~s}$ (Wang et al. 2000). Historic light curves in the X-ray, UV, and optical bands indicate that the variability amplitude in the UV and optical (up to $70 \%$ ) is much smaller than in X-rays (up to a factor of 10). The optical micro-variability of this object can actually be driven by re-processing of the variable X-ray flux if only half of the absorbed X-rays are re-radiated in the opticalto-UV band. The comparison with Einstein (Kriss 1988) and EXOSAT (Malaguti et al. 1994) observations further showed that the quasar can be classified as X-ray weak only in one out of five X-ray observations (for details see Wang et al. 2000).

To what extent the intensity variations are related to spectral changes is difficult to examine in detail due to the poor statistics of the pointed ROSAT/ASCA spectra, especially if significant X-ray scattering is present. Corbin \& Boroson (1996) mention that the quasar shows "associated absorption" in an HST spectrum and Wang et al. (2000) claim the existence of narrow associated Ly $\alpha$ absorption. There are no HST data for the C IV region, but co-added IUE data suggest C IV absorption consistent in velocity with the $\operatorname{Ly} \alpha$ absorption.

\section{The XMM-Newton observation}

PG $0844+349$ was observed by XMM-Newton on November 5, 2000. The EPIC PN was operated in Full Window mode with medium filter with a total exposure of $\sim 21 \mathrm{ksec}$. Both MOS cameras were in Large Window mode with medium filters as well, each yielding $\sim 23.5 \mathrm{ksec}$ of data. The two RGS chains were operated in Spectroscopy mode, both with exposures of $\sim 26 \mathrm{ksec}$. The OM camera was operated in Imaging mode and five images were taken with the $U$-band filter $(300-400 \mathrm{~nm})$. For the data analysis we used the XMMSAS version 5.2.0 for EPIC and OM, and version 5.3.0 for RGS.

\subsection{The light curve}

We determined the $0.5-10 \mathrm{keV}$ PN light curve of PG $0844+349$ by accumulating the photons in $400 \mathrm{~s}$ bins from a circular region of radius $r=100^{\prime \prime}$ around the quasar position; for this we selected single and double events with quality flag $=0$ (for details of the EPIC detectors see Ehle et al. 2001). For the MOS data we took singles and doubles (Pattern 0-12) as well and selected the photons from a circular region of radius $r=60^{\prime \prime}$ around the source.

During the observation the background was rather noisy with some strong flares. The total background count rate generally contributes only about $4 \%$ of the source count rate. However, in two flares the background count rate at energies above $3 \mathrm{keV}$ reaches up to $40 \%$ of the source count rate and care has to be taken in the spectral analysis. In particular, we ignore data from the last $2 \mathrm{ksec}$ of the observation as the background count rate becomes very variable then. Since there are no differences between PN and MOS light curves, in order to increase the signal-to-noise ratio, we summed up the PN count rates (average $6.61 \mathrm{cts} / \mathrm{s}$ ) and the two MOS count rates (average MOS 1: $1.93 \mathrm{cts} / \mathrm{s}$; MOS 2: $1.94 \mathrm{cts} / \mathrm{s}$ ). The summed, background subtracted, EPIC light curve shown in Fig. 1 starts at the switch on of the PN camera. There are about $2.5 \mathrm{ksec}$ of

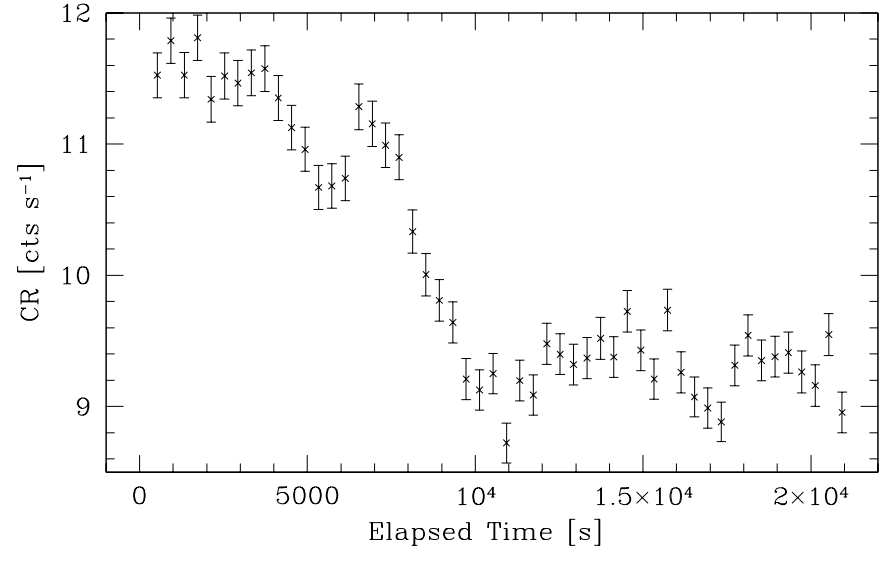

Fig. 1. Background subtracted summed PN + MOS light curve of PG $0844+349$; the time binning is $400 \mathrm{~s}$.

MOS data previous to that, where the source stays on the same high, flat count rate level. The RGS observation started around the same time as the PN, and lasted some $1.8 \mathrm{ksec}$ longer, well into the period of flaring background.

The EPIC count rate (see Fig. 1) starts to decrease after about $4 \mathrm{ksec}$, recovers for $\sim 2 \mathrm{ksec}$ and then changes by about $25 \%$ to a lower intensity level near the middle of the observation. The flux drops occur in a smooth, nearly linear fashion at a rate of $\sim 6.1 \times 10^{-4} \mathrm{cts} \mathrm{s}^{-2}$. We will show in the next section that these changes are achromatic to high statistical significance. With the spectral information obtained below, the K-corrected $0.5-10 \mathrm{keV}$ luminosity during the first part of the observation is $\sim 2.5 \times 10^{44} \mathrm{erg} \mathrm{s}^{-1}$. We can use the $\sim 20 \%$ decline of the count rate over $\sim 3500 \mathrm{~s}$ near the middle of the observation to estimate the lower limit of the radiative efficiency, $\eta>5 \times 10^{-43} \Delta L / \Delta t$ (Fabian 1984), and we obtain $\eta>7 \times 10^{-3}$ which is far below the theoretical limit for a Schwarzschild black hole.

The OM provided $U$-filter images integrated for $\sim 4000 \mathrm{~s}$ each. The brightness of the source, obtained from the Pipeline Processing System (PPS), stayed approximately constant at $14.00 \mathrm{mag}$; the largest deviation from this average value occured during the period $\sim 2-6 \mathrm{ksec}$ after the start of the PN exposure, during the first intensity dip, when the source was 0.01 mag fainter than the average. This is, however, only a $\sim 1.5 \sigma$ effect which implies that any variations of the optical flux are negligible compared to the changes in the X-ray band.

\subsection{Spectral properties}

For the EPIC spectral analysis we used the latest available response matrices (version 6.1) issued in December 2001. For the PN camera we extracted single and double events with quality flag $=0$ from a circular region with a radius of $45^{\prime \prime}$ around the source position. This radius includes $\sim 88 \%$ of the source photons (Ghizzardi \& Molendi 2001) but avoids the gap between the detector chips. The background was taken on the same chip at a similar offset position. With a count rate of $\gtrsim 7 \mathrm{cts} \mathrm{s}^{-1}$ in the high state the PN detector, operated in Full Window mode, showed strong indications of pile-up, clearly apparent from the XMMSAS task epatplot. We therefore discarded photons from 
the innermost $3 \times 3$ RAW pixels at the core of the point spread function from the spectral analysis. As a consequence, the normalizations of the models in the PN fits given in Table 1 are underestimating the actual photon flux. Further, we discarded the data of the last $\sim 2 \mathrm{ksec}$ of the observation, when the background was strongly flaring, and we excluded times of high background, i.e. greater than $50 \mathrm{cts} / \mathrm{s}$, from the Good Time Intervals (GTI) file. However, this last selection criterion influenced the quality of the fits only marginally. For the two MOS cameras we selected events with pattern $\leq 12$ and flag $=0$ and a similar extraction radius as for the PN; the background was taken from an area on the same chips near the source. Pile-up did not influence the spectral fits of the MOS data noticeably; therefore the full data were used.

The RGS spectra of PG $0844+349$ were accumulated by selecting counts from a region centered on the source position and enclosing $90 \%$ of the point spread function in the cross dispersion direction, and making the first order selection by including $90 \%$ of the expected CCD pulse height distribution of the source photons. The background spectrum was obtained from two spatial regions excluding $95 \%$ of the source point spread function in the cross dispersion direction and performing the same order selection as for the source.

While the ASCA high state data could be well fitted by a simple absorbed power law (Wang et al. 2000), this model does not provide an acceptable fit to the PN data from the first, high state part of the observation. With a reduced $\chi^{2}=1.42$ the fitted power law index is $\Gamma=2.57$ and the residuals clearly show that at higher energies the spectrum gets flatter. A broken power law, fixing the absorption at the Galactic value $N_{\mathrm{H}}=3.32 \times 10^{20} \mathrm{~cm}^{-2}$ (Lockman \& Savage 1995), gives an acceptable fit to the PN data with a reduced $\chi^{2}=1.03$ for 372 d.o.f. (see Table 1). Fitting the same time interval with the MOS1 and the MOS 2 data separately yielded similar acceptable fits. The fit parameters in Table 1 indicate that the two MOS spectra have nearly identical power law slopes. In both MOS cameras the residuals at the lowest energies show an $S$ shaped pattern with a maximum at $\sim 0.5 \mathrm{keV}$, while the PN fits, with generally slightly flatter power laws and a higher break energy, show systematic positive residuals at $\sim 0.6 \mathrm{keV}$.

Applying the same model to the low state, i.e. the period between 10 and $19 \mathrm{ksec}$ after the start of the observation, gave an acceptable fit with nearly identical parameters, with the same "typical" differences between the three detectors as above (see Table 1).

We repeated the fit over several other, shorter time intervals during the observation and always obtained acceptable fits with very similar parameters. We thus conclude that no statistically significant spectral variations occur during the flux changes of the source. Therefore for the subsequent fits we used the data from the full observation, again excluding the last $2 \mathrm{ksec}$ and times of high background.

Figure 2 shows the simultaneous broken power law fit to the whole data set for the PN and the combined MOS instruments. Due to the slight differences between the PN and MOS cameras, visible at $\sim 0.5-0.6 \mathrm{keV}$ and at higher energies, the quality of the fit is only moderate $\left(\chi_{\text {red }}^{2}=1.11\right.$ for 1006 d.o.f.). Adding a Gaussian iron line does not improve the fit, mainly

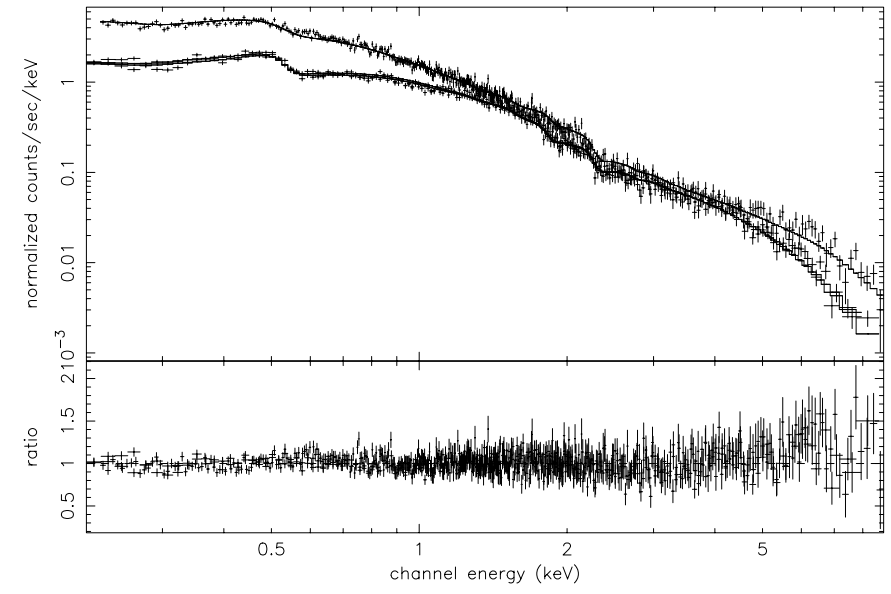

Fig. 2. Broken power law fit to the total PN + combined MOS data of PG 0844+349; the PN spectrum is the upper curve.

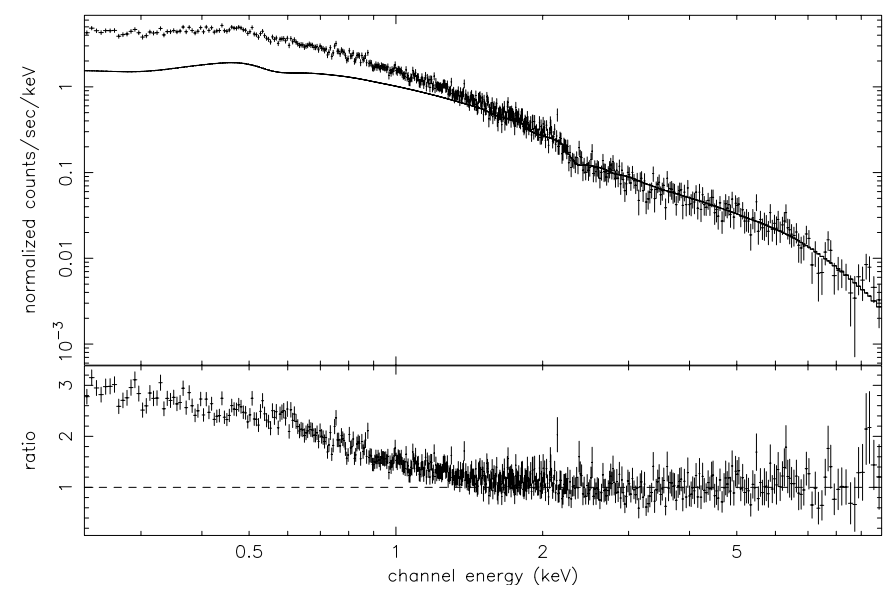

Fig. 3. Power law fit to the total PN data in the energy range 2-10 keV; the fitted model is extrapolated to lower energies. The lower panel gives the ratio between data and model.

because of the low statistical significance of the data at higher energies. We therefore fitted the PN data separately for the 2$9 \mathrm{keV}$ range with a single power law and, secondly, with a power law plus a Gaussian line. We find a broad line at an energy of $6.25 \pm 0.13 \mathrm{keV}\left(E_{1, \text { rest }}=6.65 \pm 0.13 \mathrm{keV}\right.$ in the quasar's rest frame) and an equivalent width of $\sim 235 \mathrm{eV}$. For a narrow line (i.e. fixing the Gaussian sigma at $10 \mathrm{eV}$ ) we get the same line energy and an equivalent width of $\sim 120 \mathrm{eV}$. Including the lines improves the quality of the fits marginally (see Table 1), but only at a $294 \%$ confidence level, according to an F-test. The small equivalent width found is atypical for NLSy1 galaxies, for which BeppoSax (Comastri 2000) and ASCA (Turner et al. 1998) generally find ionized iron lines with large equivalent widths (few $100 \mathrm{eV}$ ).

The moderate quality of the combined PN and MOS fits and the fit residuals can be explained as resulting from the remaining uncertainties in the detector calibrations. However, they might also indicate that the chosen spectral model can be improved.

In Fig. 3 we show the power law fit to the total PN data for the $2-10 \mathrm{keV}$ range, extrapolated to lower energies. The slope of $\Gamma \sim 2.04$, consistent with ASCA, is probably not 
Table 1. Results from spectral fitting assuming fixed Galactic $N_{\mathrm{H}}=3.32 \times 10^{20} \mathrm{~cm}^{-2}$.

\begin{tabular}{|c|c|c|c|c|c|c|c|}
\hline Period & Detector & Model & $\Gamma_{\text {soft }}$ & $\begin{array}{l}E_{\text {break }} \\
(\mathrm{keV})\end{array}$ & $\Gamma_{\text {hard }}$ & $\begin{array}{c}\text { Power law norm. } \\
\left(10^{-3} \mathrm{ph} / \mathrm{keV} / \mathrm{cm}^{2} / \mathrm{s}\right)\end{array}$ & $\chi_{\text {red }}^{2} /$ d.o.f. \\
\hline \multirow[t]{3}{*}{ High } & PN & bknpow & $2.71 \pm 0.02$ & $1.63 \pm 0.12$ & $2.13 \pm 0.06$ & $1.65 \pm 0.03$ & $1.03 / 372$ \\
\hline & MOS 1 & bknpow & $2.81 \pm 0.04$ & $1.02 \pm 0.08$ & $2.31 \pm 0.04$ & $2.96 \pm 0.12$ & $1.03 / 148$ \\
\hline & MOS 2 & bknpow & $2.85 \pm 0.04$ & $1.03 \pm 0.08$ & $2.31 \pm 0.04$ & $3.06 \pm 0.12$ & $1.10 / 145$ \\
\hline \multirow[t]{3}{*}{ Low } & PN & bknpow & $2.75 \pm 0.02$ & $1.81 \pm 0.11$ & $2.08 \pm 0.06$ & $1.32 \pm 0.02$ & $1.04 / 372$ \\
\hline & MOS 1 & bknpow & $2.77 \pm 0.03$ & $1.26 \pm 0.08$ & $2.27 \pm 0.04$ & $2.49 \pm 0.06$ & $0.92 / 167$ \\
\hline & MOS 2 & bknpow & $2.93 \pm 0.03$ & $1.05 \pm 0.06$ & $2.34 \pm 0.03$ & $2.45 \pm 0.08$ & $1.02 / 166$ \\
\hline \multirow[t]{2}{*}{ Total } & PN & bknpow & $2.72 \pm 0.01$ & $1.80 \pm 0.08$ & $2.09 \pm 0.04$ & $1.46 \pm 0.02$ & $0.98 / 537$ \\
\hline & $\mathrm{PN}+\operatorname{MOS} 1+2$ & bknpow & $2.77 \pm 0.09$ & $1.33 \pm 0.03$ & $2.25 \pm 0.02$ & (a) & $1.11 / 1006$ \\
\hline \multirow[t]{2}{*}{$\operatorname{Total}^{(1)}$} & PN & pow & & & $2.05 \pm 0.05$ & $0.96 \pm 0.10$ & $0.83 / 188$ \\
\hline & $\mathrm{PN}$ & pow + gauss & $2.11 \pm 0.05$ & $6.247 \pm 0.127^{(2)}$ & $0.24 \pm 0.16^{(3)}$ & $0.96 \pm 0.10$ & $0.81 / 185$ \\
\hline \multirow[t]{4}{*}{ Total } & $\mathrm{PN}$ & pow + bbody & & $1.94 \pm 0.14^{(4)}$ & $2.73 \pm 0.01$ & $1.45 \pm 0.02$ & $0.99 / 629$ \\
\hline & $\mathrm{PN}$ & pow + brems & & $0.29 \pm 0.01^{(4)}$ & $2.11 \pm 0.03$ & $1.02 \pm 0.06$ & $0.95 / 621$ \\
\hline & $\mathrm{PN}$ & pow + diskbb & & $0.14 \pm 0.01^{(4)}$ & $2.23 \pm 0.02$ & $1.17 \pm 0.04$ & $1.00 / 629$ \\
\hline & $\mathrm{PN}$ & pow + mekal & & $0.26 \pm 0.01^{(4)}$ & $2.18 \pm 0.03$ & $1.12 \pm 0.05$ & $0.95 / 627$ \\
\hline
\end{tabular}

(a) Normalizations for the individual detectors allowed to be different.

(1) Fit over the 2-9 keV energy band only; ${ }^{(2)}$ Fitted line energy; ${ }^{(3)}$ Line width (sigma) in keV; the photon flux in the line is $(5.7 \pm 4.9) \times 10^{-6}$ photons $/ \mathrm{cm}^{2} / \mathrm{s} .{ }^{(4)}$ Temperature of the additional model component in $\mathrm{keV}$.

affected by contributions of more complex models for the low energy part of the spectrum. The ratio between the data and the model clearly shows the "gradual soft excess" (Pounds \& Reeves 2002) also found in other AGN with the high sensitivity and large bandwidth of XMM-Newton. While the hard power law tail seems to be well constrained, the exact shape of the spectrum at low energies remains uncertain. We therefore tried several composite models, mainly to characterize the softer part of the spectrum.

Models including a thermal component in addition to the hard power law fit the data quite well, as shown in Table 1; the relative contribution of the power law component can be inferred from the listed normalization. The power law + bbody fit provides two solutions: one in which the power law component represents the hard flux, the black body temperature is similar to that of the diskbbody and another, perhaps less physical solution, where the steep power law provides the soft flux, the hot bbody the hard flux (these parameters are given in Table 1). All models are statistically acceptable (although the above mentioned systematic residuals at $\sim 0.6 \mathrm{keV}$ persist in all fits) and therefore the physical nature of the soft emission remains unclear.

As the fitted slope of the spectrum at higher energies seems to harden with energy we tried to fit a curved continuum model (Fossati et al. 2000) but no good fit could be achieved. We further tried several composite cases fixing the hard power law index at the best fit slope of the $2-10 \mathrm{keV}$ fit but we never obtained an acceptable fit: this seems to indicate that the hard power law is only the tail of a distribution and not a separate component.

Nearly featureless spectra with a strong soft component are indicative of Comptonization of soft photons in the hot corona of an accretion disk (Haardt \& Maraschi 1993). A fit with the
Xspec Comptonization model comptt (Titarchuk 1994) failed to reproduce the hard power law and resulted in an unacceptable fit $\left(\chi_{\text {red }}^{2}=1.97\right)$. Adding a hard power law to the comptt model $\left(\chi_{\text {red }}^{2}=1.09 / 1094\right.$ d.o.f. $)$ or fitting the sum of two Comptonization models with different temperatures and optical depths of the scattering medium $\left(\chi_{\text {red }}^{2}=1.11 / 1093\right.$ d.o.f. $)$ resulted in fits nearly as good as the models in Table 1 . For the double - comptt fit we either assumed the same temperature for the two soft photon components or left them free to vary independently. In all three cases the bulk of the flux up to $\sim 3 \mathrm{keV}$ originates from Comptonization of soft photons off a gas of $k T \sim 4.5 \mathrm{keV}$ and optical depth $\tau \sim 2.4$. The high energy part of the spectrum is formed either by the power law component or the second Comptonization component with $k T \sim 16 \mathrm{keV}$ and $\tau \sim 3.3$. The temperatures of the soft photons were fitted to be $k T_{0}=65_{-65}^{+99} \mathrm{eV}$ in case of the extra power law component, $k T_{0}=3_{-3}^{+71} \mathrm{eV}$ (using the same values for the two soft components) and $k T_{0}(1)=12_{-12}^{+169} \mathrm{eV}$ and $k T_{0}(2)=2_{-2}^{+341} \mathrm{eV}$ for the case of two independent soft components, respectively. The temperatures of the Comptonizing electrons are at the low end of the expected temperature range for AGN (Haardt \& Maraschi 1993) while the fitted optical depths are higher than usually deduced. The temperatures of the soft photons appear rather low; however, the parameters are very poorly constrained because of the close coupling of temperature and optical depth in the Comptonization models. Interestingly, the high optical depth implies that any reflection features (like the iron line) are suppressed by Compton scattering in the corona itself (Matt et al. 1997).

The superior energy resolution of the RGS data could provide an ultimate test for the nature of the soft emission. The same composite models tried on the PN data alone (see Table 1) were fitted simultaneously to the RGS1 and RGS2 spectra, as 


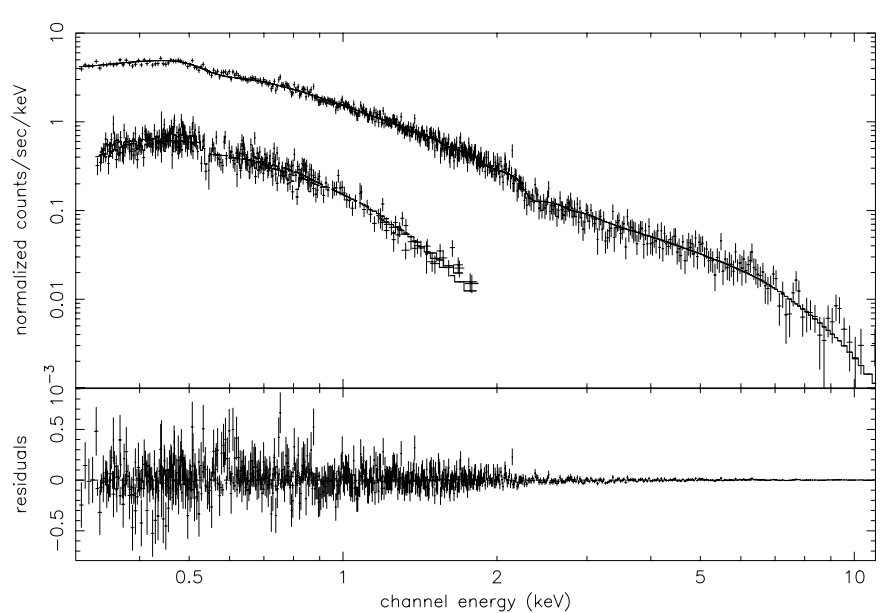

Fig. 4. Power law + bremsstrahlung fit to the PN, RGS1 and RGS2 data of PG $0844+349$

well as to the PN. The hard power law slope and the temperature of the soft component were kept fix at the best fit value obtained from the fits of the PN alone, and the normalizations for the three instruments were allowed to vary independently of each other (because of the PN data selection used to avoid pile-up). The low energy absorption column was fixed at the Galactic value. Values of $\chi_{\text {red }}^{2} /$ d.o.f. very similar to those in Table 1 for the PN alone were obtained (in the range 0.98$1.05 / 841$ ), with the formally best fit model being the combination of a power law and a bremsstrahlung component. This best fit and the data are shown in Fig. 4; the fit of the pow/diskbb and pow/mekal are virtually indistinguishable from this. Although the signal-to-noise ratio of the RGS data is only moderate, it is clear that there is no evidence of emission or absorption structures in the spectrum, indicating the absence of any additional source intrinsic absorbers. In particular, there is no evidence of excess emission in the RGS at $\sim 0.6 \mathrm{keV}$ which could explain the amplitude and the shape of the residuals observed in the PN fit.

In the following, we use the broken power law model for the determination of the source's energetics; however, it should be kept in mind that this is a rather artificial representation of the source's spectrum and that at the lowest energies the flux distribution might be quite different from a simple power law, introducing some uncertainty in the numerical values.

The observed average un-absorbed flux in the high state in the $0.2-10.0 \mathrm{keV}$ range amounts to $2.6 \times 10^{-11} \mathrm{erg} \mathrm{cm}^{-2} \mathrm{~s}^{-1}$, while the average in the low state is $2.1 \times 10^{-11} \mathrm{erg} \mathrm{cm}^{-2} \mathrm{~s}^{-1}$. With these fluxes the K-corrected X-ray luminosity of $\mathrm{PG} 0844+349$ is in the range $L_{0.2-10 \mathrm{keV}}=(4.1-4.9) \times$ $10^{44}$ erg $\mathrm{s}^{-1}$, using a Friedman cosmology with $H_{0}=$ $50 \mathrm{~km} \mathrm{~s}^{-1} \mathrm{Mpc}^{-1}$ and $q_{0}=0.5$. The average soft-band luminosity is $L_{0.5-2 \mathrm{keV}} \sim 6.4 \times 10^{43} \mathrm{erg} \mathrm{s}^{-1}$, for the hard band we find $L_{2-10 \mathrm{keV}} \sim 6 \times 10^{43} \mathrm{erg} \mathrm{s}^{-1}$. Thus PG $0844+349$ is a quasar of rather low luminosity in the X-ray band. Using the monochromatic luminosity at $2 \mathrm{keV}$ we derive an X-ray loudness $\alpha_{\text {ox }}=-0.384 \log \left(L_{2 \mathrm{keV}} / L_{2500 \AA}\right) \sim 1.58$, which is typical for average radio-quiet quasars (Yuan et al. 1998).

\section{Discussion}

Using the normalizations of the power law fits the above analysis shows that PG $0844+349$ was in a historically high state (see Table 1 of Wang et al. 2000) during the XMM-Newton observation, with an average $0.1-2.4 \mathrm{keV}$ luminosity of $\lesssim 3 \times$ $10^{44} \mathrm{erg} \mathrm{s}^{-1}$. Judging from the OM data the object was optically rather bright as well. An extrapolation of the soft power law into the optical band over-predicts the optical flux by a factor of $\sim 4$, therefore the spectrum must break between the optical and the soft X-ray band. Nevertheless, most of the power is emitted in the soft X-ray / UV band. Taking the mass estimates for the central black hole from Kaspi et al. (2000) $\left(2-3 \times 10^{7} M_{\odot}\right)$ this implies that PG $0844+349$ must be a quite efficient accretor. The optical spectrum of PG $0844+349$ resembles very much that of NLSy1 galaxies (Boroson \& Green 1992) and thus an efficient accretion flow as well as its X-ray variability would be in accordance with current models of these AGN.

The X-ray spectrum of PG $0844+349$ shows a relatively flat power law component at higher energies $\left(\Gamma_{\text {hard }} \sim 2.25\right)$ and a strong, steep soft excess at energies $\lesssim 2 \mathrm{keV}$. Both components are featureless and the inclusion of a broad or a narrow iron line around $6.7 \mathrm{keV}$ improves the fit only marginally. The soft excess can be modeled with various components resulting in fits with similar statistical significances. The RGS data do not indicate the presence of any spectral feature either. The excess of the soft flux reaches a factor of $\sim 2.5$ over the extrapolation of the hard power law.

This kind of spectrum is not unusual amongst recent measurements of X-ray spectra of bright Seyferts/quasars with XMM-Newton (O'Brien et al. 2001; Page et al. 2001; Pounds \& Reeves 2002). As a matter of fact, the spectrum of PG $0844+349$ appears to be a carbon copy of that of the NLSy 1 galaxy PKS 0558-504 (Pounds \& Reeves 2002, Fig. 1), even with respect to the "big blue bump" seen in both objects (O'Brien et al. 2001). However, we also note that the power law slope of PG $0844+349$ is slightly steeper than that of PKS 0558-504, which is radio-loud and shows correlations between X-ray brightness and hardness (Gliozzi et al. 2001), characteristics not shared by PG $0844+349$.

The featureless spectra in these objects indicate that we are seeing the bare continuum disk emission from the quasars: thus disk Comptonization models, where the X-rays are produced via inverse Compton emission in a hot corona embedding a cooler accretion disk (e.g. Haardt \& Maraschi 1993; Pounds et al. 1995), might provide a satisfactory physical explanation. Fits with available models for this scenario, however, yield parameters for PG $0844+349$ not typically found in other AGN, and not statistically preferred according to the above analysis: this might indicate that the actual physical conditions in the sources are more complex than our simplified models can account for.

The large variety of possible Comptonization scenarios (for example, Haardt 1996; Zycki et al. 2001) does not allow a better confinement of the parameters in the physical phase space. Stronger constraints might be deduceable from an analysis of the temporal behavior of the source. PG 0844+349 was known to be variable in X-rays from previous ROSAT and ASCA 
observations, but only XMM-Newton is able to follow the flux changes on the shortest time scales. The X-ray flux (Fig. 1) basically changes during the observation from a higher to a lower level in a nearly linear fashion and the slopes of the intensity variations are very similar, indicating a rather well organized process. The light curve shows (for a NLSy1) an atypically low variance and the intensity changes occur without any measurable spectral changes in the X-ray band.

The optical image of PG $0844+349$ indicates that we are seeing the object nearly pole-on and therefore heavy obscuration of the X-ray emitting region appears unlikely. The emission from a jet, changing its geometrical appearance, can very likely be ruled out as the source is definitely radio-quiet, unless we are looking directly into a purely hydrodynamically outflowing jet for which there are no other observational indications.

Achromatic flux changes can result from changes of the effective radiating area, ensuring that the spectral shape does not change noticeably. While the temperature of the cold matter does not play a significant role in the models, changes in the optical depth $\tau$ give rise to significant spectral variability (Haardt et al. 1997). Most of the theoretical investigations are, however, concentrating on the higher energy part of the AGN spectrum (see, for example, Petrucci et al. 2001), and the parameter space of relevance here is only poorly explored. In any case, changes of the radiating area are expected to happen on the dynamical time scale for Keplerian inflow, $\tau \sim 9 \times 10^{3}\left(r / R_{\mathrm{s}}\right)^{3 / 2}\left(M_{\mathrm{bh}} / 10^{7} M_{\odot}\right) \mathrm{s}$, where $R_{\mathrm{s}}$ is the Schwarzschild radius of the central black hole of mass $M_{\mathrm{bh}}$. This estimate implies a rather low mass for the central black hole in PG 0844+349 and a compact emitting region. However, strong variability of Seyferts and Galactic black hole candidates indicates that the corona cannot be a uniform, continuous medium, unless it is geometrically thin (Celotti et al. 1992); moreover, observational evidence implies that the geometry of the coronal plasma cannot be slab like, but is made up of a number of distinct active regions (Haardt et al. 1994).

In a popular class of models the corona is heated by magnetic fields which rise up buoyantly from the disk where they reconnect and release their energy in flares. The energy storage in the corona is very likely the magnetic fields (Merloni \& Fabian 2001) and the picture of the corona is that of a spread of active regions, of which only a few are large and dominate at any given time. The overall time scale of the evolution of the magnetic field configuration is expected to be of the order of the Keplerian time scale again (Romanova et al. 1998) but individual active regions can certainly evolve much more rapidly, depending of the magnetic field configuration and the flow conditions in the disk, which are largely unknown.

\section{Conclusions}

In a $20 \mathrm{ksec}$ XMM-Newton observation the X-ray transient quasar PG 0844+349 was found in a historically high X-ray state with an average $0.2-10 \mathrm{keV}$ luminosity of $L_{0.2-10 \mathrm{keV}} \sim$ $4.5 \times 10^{44} \mathrm{erg} \mathrm{s}^{-1}$. During the observation the flux of PG 0844+349 varied achromatically by $\sim 25 \%$ from a higher into a lower flux state in a very smooth manner on a time scale of a few thousand seconds.

The quasar showed a featureless spectrum which can be physically explained by Comptonization from hot electrons of the emission of an accretion disk. The rather low signal-tonoise ratio RGS spectra do not indicate the presence of any emission or absorption features either. The fitted values of the slopes of the power law type spectrum at high energies $\left(\Gamma_{\text {hard }} \sim 2.05\right)$ and those of the power law in the soft band $\left(\Gamma_{\text {soft }} \sim 2.75\right)$ differ slightly between the EPIC detectors and depend on the complexity of the models fitted to the data. Current Comptonization models predict considerably lower temperatures and higher optical depths of the Compton scattering electrons than generally found in Seyfert galaxies and radioquiet quasars (see e.g. Petrucci et al. 2001). As PG 0844+349 shares many of the characteristics of NLSy1 galaxies and similar parameters were recently deduced for other NLSy1 galaxies (O'Brien et al. 2001; Page et al. 2001) we propose that Comptonization spectra with high optical depths and moderate plasma temperatures might be a distinguishing criterion for the accretion process in NLSy1 galaxies.

More sensitive XMM-Newton observations and a deeper theoretical exploration of this lower energy and lower temperature parameter space of Comptonization models might shed some new light into the physical properties of these objects.

Acknowledgements. This research has made use of the NASA/IPAC Extragalactic Data Base (NED) which is operated by the Jet Propulsion Laboratory, California Institute of Technology, under contract with the National Aeronautics and Space Administration. This work is based on observations with XMM-Newton, an ESA science mission with instruments and contributions directly funded by ESA Member States and the USA (NASA). The Mullard Space Science Laboratory acknowledges financial support from the UK Particle Physics and Astronomy Research Council.

\section{References}

Boller, Th., Brandt, W. N., Leighly, K. M., \& Ward, M. J. 2000, Proc. of the Workshop on Observational and Theoretical Progress in the Study of NLS1 Galaxies, New Astron. Rev., 44, 381

Boroson, T. A., \& Green, R. F. 1992, ApJS, 80, 109

Celotti, A., Fabian, A. C., \& Rees, M. J. 1992, MNRAS, 255, 419

Clements, D. L. 2000, MNRAS, 311, 833

Comastri, A. 2000, New Astron. Rev., 44, 403

Condon, J. J., Gower, A. C., \& Hutchings, J. B. 1987, AJ, 93, 255

Corbin, M. R., \& Boroson, T. A. 1996, ApJS 107, 69

Ehle, M., Breitfellner, M., Dahlem, M., et al. 2001, XMM-Newton Users' Handbook,

http://xmm.vilspa.esa.es/xmm_user_support/ external/documentation/uhb_frame.shtml

Elvis, M. 2000, ApJ, 545, 63

Elvis, M., \& Wilkes, B. 1994, ApJS, 95, 1

Fabian, A. C. 1984, Phys. Scr., T7, 130

Ghizzardi, S., \& Molendi, S. 2001, Proc. of the Conf. New Visions of the X-ray Universe, ESTEC Nov. 2001

Fossati, G., Celotti, A., Chiaberge, M., et al. 2000, ApJ, 541, 166

Gliozzi, M., Brinkmann, W., O’Brien, P. T., et al. 2001, A\&A, 365, L128

Haardt, F. 1996, Proc. of the 2nd Italian National Workshop on AGN [astro-ph/9612082] 
Haardt, F., \& Maraschi, L. 1993, ApJ, 413, 507

Haardt, F., Maraschi, L., \& Ghisellini, G. 1994, ApJ, 432, L95

Haardt, F., Maraschi, L., \& Ghisellini, G. 1997, ApJ, 476, 620

Hutchings, J. B., \& Crampton, B. 1990, AJ, 99, 37

Jang, M., \& Miller, H. R. 1997, AJ, 114, 565

Kaspi, S., Smith, P. S., Netzer, H., et al. 2000, ApJ, 533, 631

Kriss, G. A. 1988, ApJ, 324, 809

Lockman, F. J., \& Savage, B. D. 1995, ApJS, 97, 1

Malaguti, G., Bassani, L., \& Caroli, E. 1994, ApJS, 94, 517

Maoz, D., Smith, P. S., Jannuzzi, B. T., Kaspi, S., \& Netzer, H. 1994, ApJ, 421, 34

Matt, G., Fabian, A. C., \& Reynolds, C. S. 1997, MNRAS, 289, 175

Merloni, A., \& Fabian, A. C. 2001, MNRAS, 321, 549

O’Brien, P. T., Reeves, J. N., Turner, M. J. L., et al. 2001, A\&A, 365, L122

Osterbrock, D. E., \& Pogge, R. W. 1985, ApJ, 297, 166

Page, K. L., Pounds, K. A., Reeves, J. N., \& O’Brien, P. T. 2002, MNRAS, 330, L1
Petrucci, P. O., Haardt, F., Maraschi, L., et al. 2001, ApJ, 556, 716

Pounds, K. A., Done, C., \& Osborne, J. 1995, MNRAS, 277, L5

Pounds, K. A., \& Reeves, J. N. 2002, Proc. of the Conf. New Visions of the X-ray Universe, ESTEC Nov. 2001 [astro-ph/0201436]

Rachen, J. P., Mannheim, K., \& Biermann, P. 1996, A\&A, 310, 371

Romanova, M. M., Ustyugova, G. V., Koldoba, A. V., Chechetkin, V. M., \& Lovelace, R. V. E. 1998, ApJ, 500, 703

Schmidt, M., \& Green, R. F. 1983, ApJ, 269, 352

Titarchuk, L. 1994, ApJ, 489, 570

Turner, T. J., George, I. M., \& Nandra, K. 1998, ApJ, 508, 648

Wang, T. G., Brinkmann, W., \& Bergeron, J. 1996, A\&A, 309, 81

Wang, T. G., Brinkmann, W., Matsuoka, M., Wang, J. X., \& Yuan, W. 2000, ApJ, 533, 113

Yuan, W., Brinkmann, W., Siebert, J., \& Voges, W. 1998, A\&A, 330, 108

Zycki, P. T., Done, C., \& Smith, D. A. 2001, MNRAS, 326, 1367 